\title{
Superstring Cosmology
}

\author{
John Estes ${ }^{* \dagger}$ \\ Laboratoire de Physique Théorique, Ecole Normale Supérieure \\ Instituut voor Theoretische Fysica, Katholieke Universiteit Leuven \\ E-mail: johnaldonestes@gmail.com
}

We review some of the recent results in the cosmology of superstrings, within the framework of string perturbation theory. We discuss a resolution of the Big Bang singularity as a bouncing cosmology via the Hagedorn transition of string theory. The era following this Hagedorn era is the intermediate era, in which the supersymmetry breaking scale, $M_{\text {susy }}$, is a dynamical quantity. The quantum and thermal corrections of the effective potential drive $M_{\text {susy }}$ to evolve proportionally to the temperature, thereby generating the desired hierarchy $M_{\text {susy }} \ll M_{\text {Planck }}$. Additionally, the quantum/thermal effective potential can be used to stabilize most of the moduli in the theory. Finally, we discuss a mechanism to decompactify spatial directions during this era.

35th International Conference of High Energy Physics

July 22-28, 2010

Paris, France

* Speaker.

$\dagger$ This work is supported from the Groupement d'Intérêt Scientifique P2I, as well as the FWO - Vlaanderen, Project No. G.0235.05, and in part by the Federal Office for Scientific, Technical and Cultural Affairs through the Interuniversity Attraction Poles Programme Belgian Science Policy P6/11-P. 
The cosmological evolution of our Universe depends not only on the known matter and interactions described at low energies, but also on all of the possible relevant degrees of freedom and interactions during the entire history of our Universe. While it is possible to describe the late time cosmological behavior by an infrared effective field theory (EFT) valid for temperatures below the electro-weak scale $M_{\mathrm{ew}}$, one may only parameterize quantities like dark energy, without explaining their origin. Moreover, the scale hierarchy $M_{\text {susy }} / M_{\text {Planck }} \ll 1$, as well as the dynamical breaking of the electro-weak gauge group need to be understood. Additionally, when one extrapolates the cosmological evolution backwards in time, one encounters the Big Bang singularity. A more fundamental theory is therefore necessary in order to explain the above infrared ambiguities appearing in the EFT.

In this review, we summarize recent progress in constructing a complete picture for the history of our Universe within the context of perturbative superstring theory. The conjectured picture is as follows. Supersymmetry is broken throughout the history of the Universe, in such a way that the supersymmetry breaking scale, $M_{\text {susy }}$, is a dynamical degree of freedom. The Big Bang singularity is resolved by a high temperature Hagedorn era, during which the Universe undergoes a bounce. ${ }^{1}$ As the Universe expands and cools, the effective potential induced by thermal and quantum fluctuations drives some of the internal directions to decompactify while stabilizing most of the remaining moduli. ${ }^{2}$ During this intermediate era, $M_{\text {susy }}$ evolves proportional to the temperature scale, with a fixed model dependent proportionality constant, while the remaining moduli settle into their minima. As the temperature falls, various infrared effects must be taken into account, which stabilize the remaining moduli. Most importantly, radiative corrections eventually lead to the spontaneous breaking of electro-weak symmetry as well as the stabilization of $M_{\text {susy }}$ at a value of the order of the electro-weak scale. At this point the standard model particles have become massive and we enter into the observed cosmological era.

In the approach considered here, one first picks an exact string background. Supersymmetry breaking is introduced at the string level by internal geometric fluxes, along with temperature. The genus one correction to the effective action is computed at the full string level. As a result of the supersymmetry breaking and temperature, the genus one correction induces an evolution of the background fields. In this approach we are assuming the back-reaction of the quantum/thermal effective potential may be treated perturbatively, which requires that we have a quasi-static evolution.

Assuming the weak or null energy condition and extrapolating the observed cosmological evolution backwards in time using the equations of quantum field theory and Einstein's theory of gravity, one finds a curvature singularity, or Big Bang, which signifies a breakdown in our description of the underlying physics. In string theory, one encounters backgrounds where the lowenergy geometric description breaks down, while the full stringy description remains valid. Thus there is hope to resolve the Big Bang singularity in terms of a stringy description.

One obstacle to this idea, is the appearance of Hagedorn instabilities in string theory at a temperature $T_{H}$. These instabilities occur as a result of the exponential growth in the number of string states as a function of mass, and signal a phase transition to a new background. Often one must include large back-reactions to the initial background. As a result, the resolution of the

\footnotetext{
${ }^{1}$ It is possible in certain cases that the bounce may be replaced by an instanton transition.

${ }^{2}$ Additional effects in the infrared, such as radiative corrections, need to be taken into account in order to stabilize the dilaton, axion and $M_{\text {susy }}$, while the remaining moduli are naturally stabilized by the thermal/quantum effective potential.
} 
Hagedorn transition often requires one to go beyond string perturbation theory, for example, by exploiting string-string dualities.

However, in recent work [1], string backgrounds in both four- and two-dimensions have been constructed which allow one to study the Hagedorn transition within the context of perturbative string theory. These models exploit a deformation of the standard canonical ensemble, in which in addition to temperature, one allows for nontrivial "gravito-magnetic" fluxes associated to the $U(1)$ graviphoton and $U(1)$ axial vector gauge fields. These fluxes describe non-dynamical gauge field condensates which have a locally vanishing field strength but a non-trivial Wilson line value around the Euclidean time circle. ${ }^{3}$ The states which are charged under these condensates are massive and effectively decouple from the system at low temperatures. Thus at low temperatures (as compared to the string scale), we recover the standard canonical ensemble.

A common feature of the two-dimensional models is a novel Massive-Spectrum-DegeneracySymmetry (MSDS). Furthermore the genus one partition function can be calculated exactly at the full string level. For the Hybrid models, one obtains

$$
\frac{Z_{\text {Hybrid }}}{V_{1}}=24\left(R_{0}+\frac{1}{2 R_{0}}\right)-24\left|R_{0}-\frac{1}{2 R_{0}}\right|
$$

where $R_{0}$ is the radius of the Euclidean time-cycle in string frame and $V_{1}$ is the volume of space. In [2], it was shown that the effective action for the Hybrid models contains, in addition to the gravitydilaton terms and thermal effective potential terms, a contribution from a space-like brane localized at the phase transition $T=T_{H}$. The brane can be understood as the localized pressure, sourced by extra massless scalars at the extended symmetry point. One finds the following cosmological behavior (in conformal gauge)

$$
d s^{2}=\frac{4}{\kappa^{2}} \frac{e^{|\tau|}}{1+|\tau|}\left(-d \tau^{2}+d x^{2}\right) \quad g_{s}^{2} \equiv e^{2 \phi(\tau)}=\frac{\pi \kappa^{2}}{192} \frac{1}{1+|\tau|}
$$

where $d s^{2}$ is the metric (in string frame) and $g_{s}$ is the string coupling. Thus instead of finding a Big Bang singularity, one finds a bouncing cosmological evolution.

We now turn to the study of the evolution in the intermediate era defined by $T_{H} \gg T \gg M_{\mathrm{ew}}$. In this era we are in four-dimensions and the temperature, $T$, has fallen well below the string scale, $T_{H}$, but remains above the electro-weak symmetry breaking scale, $M_{\mathrm{ew}}$. It is possible to study this era independently from the Hagedorn era by allowing the moduli and fields to take arbitrary initial values. One takes a well defined string background with $\mathscr{N}=1$ supersymmetry, for example heterotic theory compactified on $T^{6} /\left(\mathbb{Z}_{2} \times \mathbb{Z}_{2}\right)$. Supersymmetry breaking, $\mathscr{N}=1 \rightarrow 0$, with supersymmetry breaking scale, $M_{\text {susy }}$, is implemented by internal "geometrical fluxes", which may be treated in string perturbation theory. ${ }^{4}$ In the simplest models, supersymmetry is broken by only one cycle wrapped by such "geometrical fluxes". In this case, for any four-dimensional

\footnotetext{
${ }^{3} \mathrm{An}$ argument in favor that this is the correct resolution, is that the condensates give the thermal vacuum the nonzero winding and momentum numbers which one would expect from considering condensation of the tachyonic modes in the standard canonical ensemble.

${ }^{4}$ This is a stringy version of the Scherk-Schwarz mechanism and may be thought of as giving the world-sheet fields twisted boundary conditions in the internal space.
} 
toroidal orbifold, the free-energy density takes the general form [3]:

$$
\mathscr{F}=-T^{4}\left(n_{T} f_{T}(z)+\tilde{n}_{T} c_{4}+n_{V} f_{V}(z)\right)
$$

where we have introduced the ratio $e^{z} \equiv M / T$. The functions $f_{T}(z)$ and $f_{V}(z)$ are given by

$$
f_{T}(z)=\frac{\Gamma(5 / 2)}{\pi^{5 / 2}} \sum_{\tilde{k}_{0}, \tilde{k}_{4}} \frac{e^{4 z}}{\left[e^{2 z}\left(2 \tilde{k}_{0}+1\right)^{2}+\left(2 \tilde{k}_{4}\right)^{2}\right]^{5 / 2}} \quad \text { and } \quad f_{V}(z)=e^{3 z} f_{T}(-z)
$$

while the integer coefficients satisfy $n_{T}>0,-n_{T} \leq n_{V} \leq n_{T}$ and $\tilde{n}_{T} \geq 0$. When one considers additional supersymmetry breaking cycles, $\mathscr{F}$ contains additional integer contributions dressed by generalized functions $f_{T(V)}$. The functions $f_{T}(z)$ and $f_{V}(z)$ contain the contributions to the freeenergy density from the entire Kaluza-Klein tower of the supersymmetry breaking cycle. They correspond respectively to contributions from the thermal and quantum fluctuations.

The free-energy density (3) results in a non-trivial potential for the supersymmetry breaking modulus [4]. The possible behaviors are summarized as

\begin{tabular}{l|c|c|c} 
& $\frac{n_{V}}{n_{T}} \leq-\frac{1}{15}$ & $-\frac{1}{15}<\frac{n_{V}}{n_{T}}<0$ & $\frac{n_{V}}{n_{T}}>0$ \\
\hline$\tilde{n}_{T}=0$ & $R D S^{5}$ & $R L D S^{4}$ & contraction \\
$\tilde{n}_{T}>0$ & $R L D S^{4}$ & $R L D S^{4}$ & contraction
\end{tabular}

The $R L D S^{4}$ corresponds to a radiation-like evolution in four dimensions, with equation of state $\rho_{\text {tot }}=3 P_{\text {tot }}$, and $M_{\text {susy }}$ evolving proportionally to $T .{ }^{5}$ The evolution of $M_{\text {susy }}$ generates the desired hierarchy $M_{\text {susy }} \ll M_{\text {Planck }}$. The RDS ${ }^{5}$ corresponds to a radiation dominated evolution in five dimensions, where the supersymmetry breaking cycle has effectively decompactified. Starting from lower dimensional models, such as the MSDS or Hybrid models discussed above, it would be interesting to use this effect to dynamically generate spatial dimensions after (or during) the Hagedorn era.

In the above discussion, we have suppressed the dependence of $\mathscr{F}$ on the remaining moduli. Including the dependence on one of the radii moduli for example, one can connect three different effective field theories [5]. For large values of the radius, the effective field theory is described by string theory in five dimensions (one of which is compact), while for small values of the radius, one must use the T-dual theory. For values of the radius close to the self dual value, the effective theory is described by four-dimensional gravity coupled to an $S U(2)$ gauge theory. String theory connects these three effective fields theories together, curing the curvature singularities which appear in the gravity theories. It turns out that the final attraction is always to a four dimensional evolution. Furthermore, there is a potential for the radius, stabilizing it at its self-dual value with a mass of order $M_{\text {susy }}$. By considering extended symmetry points, one may stabilize all the moduli describing the internal space, while the dilaton and axion require the inclusion of infrared and possibly nonperturbative effects.

To go further, one must include infrared effects, such as radiative corrections or strong coupling behavior leading to confinement in various sectors of the theory. These effects will be model

\footnotetext{
${ }^{5}$ To see it is not a purely radiation evolution, note that $\rho_{\text {thermal }}=4 P_{\text {thermal }}$. It is only after including the evolution of $M_{\text {susy }}$ that we recover $\rho_{\text {tot }}=3 P_{\text {tot }}$.
} 
dependent, while the above results in the intermediate era are robust. Perhaps most important, is the study of the electro-weak phase transition where the supersymmetry breaking scale, $M_{\text {susy }}$, is stabilized. It would be interesting to carry this out at the full string level. One output of such an investigation will allow one to study dark energy, since $M_{\text {susy }}$ will now be stabilized and will generate a cosmological constant. However, generically one would expect this value to be of order $M_{\text {susy }}^{4}$, which is too large to be compatible with observations. Additionally, there remains work in exploring the MSDS and Hybrid models of the Hagedorn era, for example, finding Hybrid vacua which naturally decompactify to four dimensions.

\section{References}

[1] I. Florakis, C. Kounnas and N. Toumbas, Nucl. Phys. B 834 (2010) 273 [arXiv:1002.2427 [hep-th]]. I. Florakis and C. Kounnas, Nucl. Phys. B 820 (2009) 237 [arXiv:0901.3055 [hep-th]].

C. Angelantonj, C. Kounnas, H. Partouche and N. Toumbas, Nucl. Phys. B 809 (2009) 291 [arXiv:0808.1357 [hep-th]]. C. Kounnas, Fortsch. Phys. 56 (2008) 1143 [arXiv:0808.1340 [hep-th]].

[2] I. Florakis, C. Kounnas, H. Partouche and N. Toumbas, Nucl. Phys. B 844 (2011) 89 [arXiv:1008.5129 [hep-th]].

[3] J. Estes, C. Kounnas and H. Partouche, arXiv:1003.0471 [hep-th].

[4] F. Bourliot, C. Kounnas and H. Partouche, Nucl. Phys. B 816 (2009) 227 [arXiv:0902.1892 [hep-th]]. T. Catelin-Jullien, C. Kounnas, H. Partouche and N. Toumbas, Nucl. Phys. B 820 (2009) 290 [arXiv:0901.0259 [hep-th]]. T. Catelin-Jullien, C. Kounnas, H. Partouche and N. Toumbas, Nucl. Phys. B 797 (2008) 137 [arXiv:0710.3895 [hep-th]].

[5] F. Bourliot, J. Estes, C. Kounnas and H. Partouche, Nucl. Phys. B 830 (2010) 330 [arXiv:0908.1881 [hep-th]]. 